\title{
Fatal Pulmonary Tumour Thrombotic Microangiopathy (PTTM) Associated with Signet Ring Cell Carcinoma of Colon: An Autopsy Diagnosis
}

\author{
Vishwakarma S, Arulselvi S*, Singh J" and Behra \\ C $^{\#}$ \\ Department of Forensic Medicine, AIIMS, India \\ "Authors are equally contributed \\ *Corresponding author: Arulselvi Subramanian, \\ Department of Lab Medicine, J ai Prakash Narayan Apex \\ Trauma Centre, AIIMS, New Delhi 110029, India
}

Received: June 03, 2017; Accepted: June 23, 2017; Published: July 10, 2017

\begin{abstract}
Pulmonary tumor thrombotic microangiopathy (PTTM) is a rare clinicopathological entity in which the tumor cells embolize to the pulmonary vasculature leading to fibro cellular intimal thickening and arteriolar occlusion by cellular intimal proliferation. Sub-acute respiratory failure, pulmonary hypertension, right sided heart failure and sudden death may be seen due to consequences of stenosis of blood vessels.

We describe a case of a 23 year old man who presented with alleged history of found unconscious and declared brought death. He was clinically diagnosed as refectory sepsis with ventilator associated pneumonia with acute kidney injury. Past history revealed Crohn's disease and treatment with Azathioprine and prednisolone. Mucicarmine was positive in colonic tumor as well as in pulmonary tumor cell emboli with recanalization and intimal fibro cellular proliferation of small arteries. A postmortem diagnosis of poorly differentiated signet ring cell carcinoma of colon with PTTM was made based on autopsy results.

Unfortunately, PTTM is difficult to diagnose and is mostly a post mortem diagnosis with an extremely poor prognosis. Pulmonary hypertension due to metastatic tumor emboli should be included in the differential diagnosis of various causes of dyspnea in patients with cancer.
\end{abstract}

Keywords: Pulmonary tumor thrombotic microangiopathy; Cell carcinoma

\section{Introduction}

Pulmonary tumor thrombotic microangiopathy (PTTM) is a rare clinicopathological entity in which the tumor cells embolize, organize and recanalize to the pulmonary vasculature leading to fibro cellular intimal thickening and vessel stenosis. Sub-acute respiratory failure, pulmonary hypertension, right sided heart failure and sudden death may be seen due to consequences of stenosis of blood vessels caused by PTTM. In this report, we describe a rare case of PTTM associated with metastatic colon carcinoma diagnosed on postmortem examination of a young man. We also aim to review the literature related to PTTM and associated malignancies and how they were diagnosed and managed.

\section{Case Presentation}

\section{Clinical course}

The deceased, a 23 year old man was brought with alleged h/o unconsciousness for an hour in a public place. On examination, patient was breathless and gasping. His BP was 110/60 mm Hg and pulse was feeble. He was immediately started on oxygen inhalation and nebulization and shifted to ICU. In ICU he was put on ventilator with i/v antibiotics and symptomatic care. On systemic examination respiratory system showed only gasping efforts and cardiovascular system showed feeble heart sounds. On CNS examination, Glasgow coma scale was E1V1M1. There was history of fever and breathlessness since past 3-4 days along with greenish expectoration. He was clinically diagnosed and treated as a case of refractory sepsis with septic shock, ventilator associated pneumonia and acute kidney injury.

\section{Previous special investigations}

His previous records revealed complaints of pain in abdomen and increased frequency of stool admixed with blood off and on, two years back. Colonoscopy revealed a long serpiginous ulcer present in transverse colon and impression of-colonic disease was made. Biopsy taken from recto sigmoid region was reported as scanty tissue with nonspecific acute on chronic inflammation. Contrast enhance CT was reported as diffuse colitis with rectal involvement with loss of haustrations and shortening of colon suggestive of ulcerative colitis. USG whole abdomen revealed slightly hypoechoic liver, mild fatty infiltration of pancreas, swollen tip of pancreas but no periappendiceal mass or fluid collection. Diffuse concentric thickening of colonic walls was seen. His routine blood investigations done twice during this period were within normal limits. Therefore he was considered as a case of inflammatory bowel disease (IBD) and since last two year she was on treatment with Azathioprine and prednisolone. He had poor compliance to medicines and was lost to follow up for last 8 months. During hospital stay his condition deteriorated further and on fifth day of admission, despite all our efforts the patient could not be revived and was declared dead. 


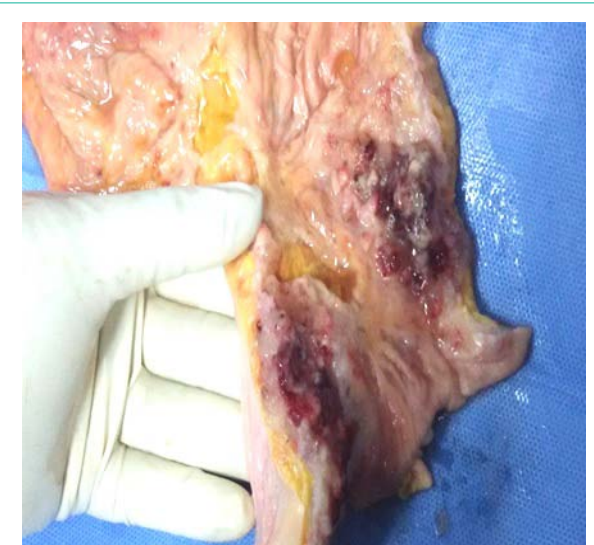

Figure 1: Gross examination: cut surface of large intestinal mucosa showing red brown ulcerated areas with thickened ulcer margin.

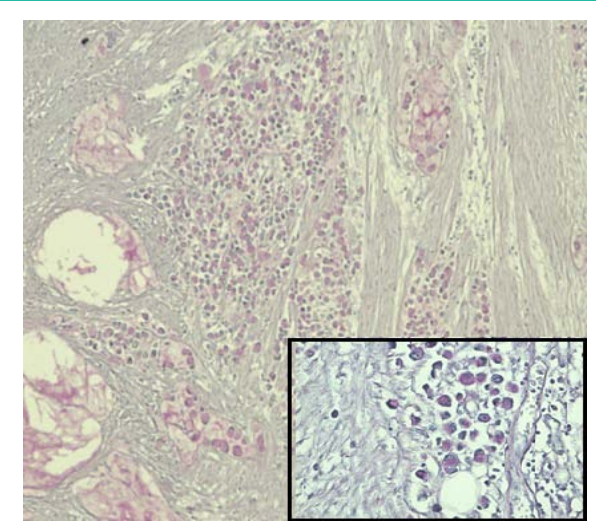

Figure 2: 4 x Microphotograph- Large intestine showing poorly differentiated malignant cells invading through the muscularis mucosa into the subserosa and extracellular mucin (Insert $10 \mathrm{x}$ signet ring cells, both mucicarmine stain.

\section{On Autopsy}

External examination: The deceased was averagely built and averagely nourished. Rigor mortis had set in and postmortem lividity present. Both corneas were hazy; pupils were dilated and fixed. On examining natural orifices- NAD (no abnormality detected).

Internal examination: Head \& Neck: was normal, Scalp \& Vault of Skull - Showed no gross abnormalities. Meninges - The dura appeared normal. There was no evidence of venous sinuses thrombosis. There was no subdural/subarachnoid hemorrhage. Brain weighed 1400 gms. There was no evidence of any hemorrhage externally or on cut surface of brain. There was no midline shift or herniation.

Thorax: Pleura and pleural cavity: The pleural cavities showed occasional flimsy adhesions with approximately $75-100 \mathrm{ml}$ of straw colored fluid in both pleural cavities. Larynx, trachea and bronchi were within normal limits. Few small paratracheal lymph nodes were noted. Lungs - Right lung weighed 715 grams and left lung weighted 610 grams. Both lungs appeared boggy and subcrepitant. Pericardium \& Pericardial cavity appeared unremarkable. Heart weighed 350 grams. The inflow/out flow tracts, valves and papillary muscles were normal. The right and left ventricular walls were normal. Coronary Arteries Both right and left coronaries were patent. No atheromatous lesions were seen. The great vessels were unremarkable.

Abdomen: Peritoneum and peritoneal cavity had $600 \mathrm{ml}$ thin straw color peritoneal fluid multiple adhesions and palpable nodularity at places. Stomach - mucosa was diffusely hemorrhagic with flattened mucosal roughage. Serosal surface was grossly unremarkable. Large Intestine had focal red black colored ulcerated mucosa. External surface of small and large intestine was edematous and congested at places. Intestinal was focally thickened with polypoid areas causing narrowing of intestinal lumen in small intestine, large intestine and rectum (Figure 1). Rectal lumen was narrowed and thickened $(1.5 \mathrm{~cm})$ at places. Rectal mucosa was reddish black with presence of mucosal ulcer ranging from $0.5 \mathrm{~cm}$ to $2 \mathrm{~cm}$. Liver, Gall Bladder and Bile Ducts - Liver weighed 1500 grams. The external surface was smooth. Cut surface was mildly congested. The Gallbladder and bile duct were normal. The hepatobiliary passage was patent. Pancreas was unremarkable. Kidneys - The left and right kidneys weighed 250 gms each. Capsule could be stripped off easily. The corticomedullary junction was well defined. Urinary Tract was within normal limits. Spleen - weighed 150 grams and cut surface appeared congested. Both adrenals appeared normal and their cut surfaces were unremarkable.

Salient gross autopsy findings were heavy, boggy, subcrepitant lungs, focal ulcerated intestinal mucosa with focal nodularities in intestinal wall.

Histopathological examination: Ulcerated and nodular areas of large intestine confirmed a tissue diagnosis of poorly differentiated mucinous adenocarcinoma of colon invading through the muscularis propria into the subserosa (Figure 2) Both lungs revealed similar tumor cells embolized, organized and recanalized in the pulmonary vasculature leading to mild fibro cellular intimal thickening and luminal narrowing with areas of intra-luminal thrombosis consistent with PTTM. The tumor cells were identical in histological appearance to that of the primary mucinous signet ring cell carcinoma of the colon found on autopsy (Figure 3). Special stain mucicarmine was positive in colonic tumor as well as in pulmonary tumor cell emboli. (TNM stage T3).

The liver showed normal lobular architecture. Some sinusoids were dilated with mild centrilobular congestion. Spleen: showed normal architecture with congestion of red pulp. Sections from both

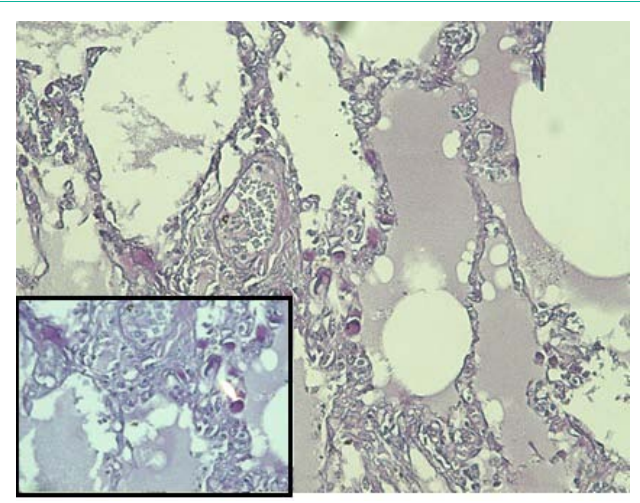

Figure 3: $10 \mathrm{x}$ Microphotograph Pulmonary vessels showing small vessels with presence of scattered signet ring tumor cells. (Insert 40x, both mucicarmine stain). 
kidneys show normal glomeruli. There is no thickening of basement membrane, the tubules and interstitium were showing early changes of acute tubular necrosis. Blood vessels were unremarkable. Sections taken from heart (right and left ventricles) showed a normal epicardial lining with unremarkable myocardial musculature. Sections from right coronary, left coronaries and left circumflex arteries are unremarkable. No atheromatous plaques seen. Stomach, Pancreas and Gall Bladder sections were unremarkable.

\section{Cause of Death}

On autopsy, deceased was found to have established diagnosis of poorly differentiated signet ring cell carcinoma of colon with features of PTTM (Stage T3NxM1).

\section{Review of literature and discussion}

First defined by Von Herbay, et al., in 1990, PTTM is known as a rare but fatal pulmonary complication seen associated with various malignancies [1]. Von Herbay in a retrospective study involving examination of 630 autopsy cases of death associated with metastatic carcinoma found that PTTM was found in $3.3 \%$ of cases, and majority of them around $90 \%$ were associated with gastric adenocarcinoma [1]. Uruga $\mathrm{H}$, et al. in a retrospective study of autopsy series of cancer death found a 1.4\% prevalence of PTTM [2]. In review of all published cases, Uruga $\mathrm{H}$, et al. found that gastric cancer accounts for $60 \%$ of cases, with others including lung cancer (9\%), cancer of unknown primary $(6 \%)$, breast cancer (3\%), and a few rarely-reported tumor sites [2]. Majority of PTTM cases have been reported from Japan may be due to the high prevalence of gastric cancer in Japan.

Clinical features noted in patients with PTTM may present as progressive dyspnea, sub-acute respiratory failure with pulmonary hypertension rapidly progressive right sided heart failure and sudden death [3]. Various malignancies from different site of origin including esophageal squamous cell carcinoma, ovarian tumors, desmoplastic small cell tumor, salivary duct carcinoma ex pleomorphic adenoma and pulmonary adenocarcinoma were also reported in literature associated with PTTM [4-10].

As antemortem diagnosis is very difficult to clinch due to the rapid development of pulmonary thromboembolism, pulmonary hypertension, heart failure and death, it is usually diagnosed on autopsy. Typically there is diagnosis of a metastatic disease at the time of presentation of PTTM [11], but occult cancer presented as PTTM can be noted albeit rarely, as found in our case.

The proposed mechanism of PTTM is that tumor cells first metastasize to the pulmonary vessels and then activate the coagulation systems and release inflammatory mediators and growth factors, causing deposition of platelets and fibrin along the intima of the arterial wall which leads to thrombosis, fibro cellular intimal proliferation, and stenosis of vessels lumen [12]. Elevated serum levels of D-dimer and fibrin degradation products, tissue factor, osteopontin and vascular endothelial growth factor (VEGF) expressed by tumor cells in addition to activated alveolar macrophages in the PTTM lesion, are believed to have an important role in onset and progression of PTTM [12-16]. Miyano, et al. reported an ante mortem known case of previously resected gastric cancer in which PTTM was suggested by increase in serum VEGF and D-dimer levels and was confirmed via transbronchial lung biopsy and video-assisted thoracoscopic surgery.
They advocated that accurate and timely diagnosis of PTTM can led to the successful treatment of the patient with use of corticosteroids, anticoagulation, and chemotherapy [12]. VEGF is believed to be a primary mediator of this process and is often reported positive in histology of PTTM; but, it is not taken as pathognomonic and should not be considered in isolation for diagnosis [13]. Clinically, patients with PTTM can show rapidly worsening respiratory function, with or without pulmonary hypertension and can also develop disseminated intravascular coagulation (DIC). In our patient acute and rapidly worsening respiratory insufficiency was noted but DIC was not found.

Various investigations viz, radiological, perfusion scan, lung biopsy and pulmonary fluid cytology are reported in literature which can be very useful to diagnose PTTM. Various CT scan findings of PTTM include multiple consolidations, ground-glass opacities, small nodular opacities, and tree-in-bud appearance in peribronchiolar areas. [17-20], but these findings are nonspecific because a "treein-bud" pattern can be seen in patients of pulmonary tumor embolism, infectious bronchiolitis and cellulose granulomatosis also. The proximal pulmonary arteries do not show any defects on contrast-enhanced CT scans of patients with PTTM, unlike tumor emboli showing them. Lung scintigraphy, characteristically shows multiple subsegmental mismatched perfusion defects in PTTM and numerous, symmetric, and more peripheral perfusion defects in tumor emboli [21]. Lung biopsy and pulmonary microvascular cytological examination using a Swan-Ganz catheter can be useful to diagnose PTTM and tumor emboli. [22]. The salient histopathological findings of PTTM which distinct it from tumor emboli are described as presence of diffuse intimal myofibroblast proliferation in pulmonary vessels and multiple microthrombi, which form due to invasion of the pulmonary vasculature by cancer cells [23]. However, rapid and serious deterioration of respiratory status often prevents performing an invasive tests viz, biopsy. In fact, a biopsy could not be performed for our case because his respiratory condition was rapidly deteriorating. Masson, et al. reported that pulmonary microvascular cytology was more convenient and less invasive test in comparison to transbronchial biopsy they reported the sensitivity and specificity of cytology as $80-88 \%$ and $82-94 \%$, respectively, in patients with lymphangitic carcinomatosis and pulmonary microvascular tumor embolization [24].

For management of these PTTM patients, chemotherapy might be the only useful treatment, but it is still debatable to use. To our knowledge, there have been only two cases that were successfully treated; one patient was treated with chemotherapy only [21]. The other was treated with corticosteroids, anti-coagulants and an anticancer drug [14]. New drugs for the treatment of pulmonary arterial hypertension, such as endothelin antagonists, prostacyclin analogues, phosphodiesterase type 5 inhibitors, and serotonin antagonists are controversial for the treatment of PTTM $[25,26]$.

During review of literature, we found 10 patients having antemortem diagnosis of PTTM $[4,17,18,27]$. The primary site of tumor was upper gastrointestinal tract in 4 of them, breasts in 3 of them, unknown in two of them, and lungs in one of them. The diagnostic methods for PTTM were transbronchial lung biopsy in five patients $[4,15,16,28,29]$, Video assisted thoracoscopic surgery in two of them [12,30], CT-guided biopsy [31] in one of them and rest two were presumptive ante mortem diagnoses based on the clinically 
proven pulmonary hypertension and presence of tumor cells obtained from wedged pulmonary artery catheter aspiration $[32,33]$.

Our case is a rare case of PTTM presented in a postmortem examination of a primary colon carcinoma. Tissue biopsy would have been an important tool to clinch the diagnosis in our case but we were not able to perform any invasive tests and cytology/biopsy tests due to rapid deterioration of the patient and due to other co morbidities misleading the diagnosis of PTTM therefore we suggest, when echocardiography indicates pulmonary hypertension despite slight and nonspecific CT findings, PTTM should be suspected, and random transbronchial lung biopsy should be sought to obtain a definitive diagnosis. Due to the rapid progressive course of PTTM, few cases have been reported where systemic chemotherapy has improved the prognosis [34]. No treatment was possible in this current case, due to the patients' rapid deterioration and lack of definitive evidence of PTTM. However, our case showed rapid deterioration in spite of administration of corticosteroid so further study is also required to assess the treatment modalities for PTTM.

\section{Conclusion}

Unfortunately, PTTM is a rapidly progressive and rare disease with poor prognosis which is caused by consequences of fatal complication of malignancy. It is a diagnostic challenge where high clinical suspicion in combination with radiological tests, histology and pulmonary artery blood sampling can lead to the correct diagnosis. It should be included in the differential diagnosis of various causes of dyspnea of unknown origin, pulmonary hypertension and sub-acute respiratory failure, where there is no improvement in respiratory symptoms with steroid therapy because the early diagnosis and treatment of PTTM may prolong the survival period of PTTM patients.

\section{References}

1. Von Herbay A, Illes A, Waldherr R, Otto HF. Pulmonary tumor thrombotic microangiopathy with pulmonary hypertension. Cancer. 1990; 66: 587-592.

2. Uruga H, Fujii T, Kurosaki A, Hanada S, Takaya H, Miyamoto A, et al Pulmonary tumor thrombotic microangiopathy: a clinical analysis of 30 autopsy cases. Intern Med. 2013; 52: 1317-1323.

3. Sakashita N, Yokose C, Fujii K, Matsumoto M, Ohnishi K, Takeya M Pulmonary tumor thrombotic microangiopathy resulting from metastatic signet ring cell carcinoma of the stomach. Pathol Int. 2007; 57: 383-387.

4. Ueda A, Fuse N, Fujii S, Sasaki T, Sugiyama J, Kojima T, et al. Pulmonary tumor thrombotic microangiopathy associated with esopahageal squamous cell carcinoma. Inter Med. 2011; 50: 2807-2810.

5. Gru AA, Pai RK, Roma AA. Pulmonary tumor thrombotic microangiopathy in patients with low grade ovarian serous neoplasm: a clinicopathologic review of 2 cases a previously unknown assocition. Int J Gynecol Pathol. 2012; 31: 438-442.

6. Sadimin ET, Collier AG, Gaffney JW, Fyfe B. Pulmonary tumor thrombotic microangiopathy with cor pulmonale due to desmoplastic small round cell tumor. J Pediatr. 2012; 160: 697-699.

7. Sekiyama T, Mizamura K, Kobayashi T, Hayashi S, Tsujino I, Hashimoto S A pulmonary tumor embolism which mimicked pulmonary tumor thrombotic microangiopathy caused by cervical cancer. Nihon Kokyuki Gakkai Zasshi. 2010; 48: 595-599.

8. Uruga $H$, Fujii T, Kurosaki A, Hanada $S$, Takaya $H$, Miyamoto A, et al. A case of pulmonary tumor thrombotic caused by carcinoma (salivary duct carcinoma) ex pleomorphic adenoma. Nihon Kokyuki Gakkai Zasshi. 2010; 48: 463-468.
9. Malani AK, Gupta C, Kutty AV, Betlej T. Pulmonary tumor thrombotic microangiopathy from metastatic gallbladder carcinoma: an unusual cause of severe pulmonary hypertension. Dig Dis Sci. 2007; 52: 555-557.

10. Hotta M, Ishida M, Kojima F, Iwai Y, Okabe H. Pulmonary tumor thrombotic microangiopathy caused by lung adenocarcinoma: Case report with review of the literature. Oncol Lett. 2011; 2: 435-437.

11. Mandalyia R, Farhat S, Uprety D, Balla M, Gandhi A, Goldhahn R, et al. Occult gastric cancer presenting as hypoxia from pulmonary tumor thrombotic microangiopathy. J Gastric Cancer. 2014; 14: 142-146.

12. Miyano S, Izumi S, Takeda Y, Tokuhara M, Mochizuki M, Matsubara O, et al. Pulmonary tumor thrombotic microangiopathy. J Clin Oncol. 2007; 25: 597599.

13. Kuwabara H, Yoshida S, Takasu T, Yuki M, Goto I, Hanafusa T, et al. Pulmonary tumor thrombotic microangiopathy caused by gastric cancer. Ann Th orac Med. 2012; 7: 168-169.

14. Chinen K, Fujino T, Horita A, Sakamoto A, Fujioka Y. Pulmonary tumor thrombotic microangiopathy caused by an ovarian cancer expressing tissue factor and vascular endothelial growth factor. Pathol Res Pract. 2009; 205: 63-68.

15. Okubo Y, Nakayama M, Kitahara K, Nemoto T, Yokose T, Abeet F, et al. Pulmonary tumor thrombotic microangiopathy induced by gastric carcinoma: Morphometric and immunohistochemical analysis of six autopsy cases. Diagnostic Pathology. 2011; 6: 27.

16. Yokomine T, Hirakawa H, Ozawa E, Shibata K, Nakayama T. Pulmonary thrombotic microangiopathy caused by gastric carcinoma. J Clin Pathol. 2010; 63: 367-369.

17. Ishiguro T, Takayanagi N, Ando M, Yanagisawa T, Shimizu Y, Sugita Y. Pulmonary tumor thrombotic microangiopathy responding to chemotherapy. Nihon Kokyuki Gakkai Zasshi. 2011; 49: 681-687.

18. Noguchi S, Imanaga T, Shimizu M, Nakano T, Miyazaki N. A case of pulmonary tumor thrombotic microangiopathy diagnosed by transbronchial lung biopsy. Nihon Kokyuki Gakkai Zasshi. 2008; 46: 493-496.

19. Shepard JA, Moore EH, Templeton PA, McLoud TC. Pulmonary intravascular tumor emboli: dilated and beaded peripheral pulmonary arteries at CT. Radiology. 1993; 187: 797-801.

20. Franquet T, Gim'enez A, Prats R, Rodr'iguez-Arias JM, Rodriguez C. Thrombotic microangiopathy of pulmonary tumors: a vascular cause of treein-bud pattern on CT. The American Journal of Roentgenology. 2002; 179: 897-899.

21. Chen WL, Cherng SC, Hwang WS, Wang DJ, Wei J. Perfusion scan in pulmonary tumor microembolism: report of a case. J Formos Med Assoc. 1991; 90: 863-866.

22. Goldhaber SZ, Dricker E, Buring JE, Eberlein K, Godleski JJ, Mayer RJ, et al. Clinical suspicion of autopsy-proven thrombotic and tumor pulmonary embolism in cancer patients. Am Heart J. 1987; 114: 1432-1435.

23. Chinen K, Tokuda Y, Fujiwara M, Fujioka Y. Pulmonary tumor thrombotic microangiopathy in patients with gastric carcinoma: an analysis of 6 autopsy cases and review of the literature. Pathol Res Pract. 2010; 206: 682-689.

24. Masson RG, Krikorian J, Lukl P, Evans GL, McGrath JM. Pulmonary microvascular cytology in the diagnosis of lymphangitic carcinomatosis. $\mathrm{N}$ Engl J Med. 1989; 321: 71-67.

25. Keenan NG, Nicholson AG, Oldershaw PJ. Fatal acute pulmonary hypertension caused by pulmonary tumour thrombotic microangiopathy. Int J Cardiol. 2008; 124: e11-e13.

26. Takahashi F, Kumasaka T, Nagaoka T, Wakiya M, Fujii H, Shimizut K, et al. Osteopontin expression in pulmonary tumor thrombotic microangiopathy caused by gastric carcinoma. Pathol Int. 2009; 59: 752-756.

27. Higo K, Kubota K, Takeda A, Higashi M, Ohishi M. Successful an temorten diagnosis and treatment of pulmonary tumor thrombotic microangiopathy. Intern Med. 2014; 53: 2595-2599.

28. Ogawa A, Yamadori I, Matsubara O, Matsubara H. Pulmonary tumor 
thrombotic microangiopathy with circulatory failure treated with imatinib. Intern Med. 2013; 52: 1927-1930.

29. Kitamura A, Nishimura N, Jinta T, Suda R, Yamano Y, Ishikawa G, et al A case of pulmonary tumor thrombotic microangiopathy diagnosed by transbronchial lung biopsy and treated with chemotherapy and long-term oxygen and anticoagulation therapies. Case Rep Pulmonol. 2013; 2013: 259080 .

30. Kayatani H, Matsuo K, Ueda Y, Matsushita M, Fujiwara K, Yonei T, et al. Pulmonary tumor thrombotic microangiopathy diagnosed antemortem and treated with combination chemotherapy. Intern Med. 2012; 51: 2767-27670.

31. Uruga $H$, Morokawa N, Enomoto T, Takaya H, Miyamoto A, Kishi K, et al. A case of pulmonary tumor thrombotic microangiopathy associated with lung adenocarcinoma diagnosed by CT-guided lung biopsy. Nihon Kokyuki Gakkai Zasshi. 2008; 46: 928-933.
32. Fukada I, Araki K, Minatsuki S, Fujino T, Hatano M, Numakura S, et al Imatinib Alleviated Pulmonary Hypertension Caused by Pulmonary Tumor Thrombotic Microangiopathy in a Patient With Metastatic Breast Cancer. Clin Breast Cancer. 2015; 15: e167-e170.

33. Higo K, Kubota K, Takeda A, Higashi M, Ohishi M. Successful antemortem diagnosis and treatment of pulmonary tumor thrombotic microangiopathy. Intern Med. 2014; 53: 2595-2599.

34. Patrignani A, Purcaro A, Calcagnoli F, Mandolesi A, Bearzi I, Ciampani $\mathrm{N}$. Pulmonary tumor thrombotic microangiopathy: the challenge of the antemortem diagnosis. J Cardiovasc Med. 2013. 15: 828-833.
Austin J Forensic Sci Criminol - Volume 4 Issue 3 - 2017 ISSN : 2380-0801 | www.austinpublishinggroup.com Arulselvi et al. () All rights are reserved
Citation: Vishwakarma S, Arulselvi S, Singh J and Behra C. Fatal Pulmonary Tumour Thrombotic Microangiopathy (PTTM) Associated with Signet Ring Cell Carcinoma of Colon: An Autopsy Diagnosis. Austin J Forensic Sci Criminol. 2017; 4(3): 1065 